\title{
Application-oriented Network Performance Evaluation for the Internet of Things
}

\author{
http://dx.doi.org/10.3991/ijoe.v9iS7.3195 \\ Xiaobo Yan ${ }^{1}$, Feng Wang ${ }^{1}$, Liang Hu ${ }^{1}$, Kuo Zhao ${ }^{1}$, Weiqing Xiong ${ }^{2}$ \\ ${ }^{1}$ Jilin University, Changchun, Jilin, China \\ ${ }^{2}$ Harbin Institute of Technology, Harbin, Heilongjiang, China
}

\begin{abstract}
This paper addresses network performance evaluation for the Internet of Things. There are a wide variety of networks at the bottom layer of the Internet of Things, such as wireless sensor network, ad hoc network. Therefore, it is not easy for the Internet of Things to evaluate its network performance. For the heterogeneity of the Internet of Things, this paper proposes an applicationoriented network performance evaluation model. This model combines the qualitative and quantitative evaluation method, giving the four levels of network performance firstly, and proposes a new network performance evaluation indicator, which is cumulative network performance evaluation value. In this model, it gives the qualitative and quantitative analysis for the performance of the Internet of Things at the application layer, and determines the level of network performance, then calculates the network performance evaluation value and the cumulative network performance evaluation value. By analyzing the network operating data collected, the experimental results show that the network performance level and the two network performance values characterize the status of the Internet of Things accurately. These evaluation results can provide basis for decision making to optimize network configuration and improve network performance.
\end{abstract}

Index Terms - the Internet of Things, heterogeneity, application-oriented, network performance evaluation, cumulative network performance evaluation value.

\section{INTRODUCTION}

With the rapid development of key technology of the Internet of Things ( IoT ), the IoT has been used widely and commonly, such as transportation and logistics domain, healthcare domain, smart environments domain, personal and social domain, futuristic applications domain [1]. The IoT are usually divided into three layers, from bottom to top, which are perception layer, transport layer, application layer [2]. However, the IoT has a strong heterogeneity at the bottom layer [3] [4], where there are wireless sensor network (WSN) [5], ad hoc network. Therefore, it is not easy for an IoT to evaluate its network performance.

Scientific evaluation of network performance helps network administrators and users understand network operating conditions timely and accurately, and provides a basis for decision making to optimize network configuration and improve network performance. However, Network performance evaluation methods varied with the different needs and applications. Reference [6], through the network measurement, puts forward a comprehensive evaluation method based on multiple measurement indicators. By applying this method to the packet path performance evaluation and network performance evaluation, the experimental results not only reflect the packet path performance, but also reflect the performance of the network. Reference [7] defines network performance evaluation indicators, constructs a flow model, and selects the link utilization, delay and delay jitter three indicators. Lastly, it makes network performance evaluation for a multimedia network with network simulation software NS-2. Reference [8] makes normalization and quantization processing with a variety factors which affect the network performance. Reference [9] proposes a "static + dynamic" weight calculation method based on the standard deviation of the correlation variable weight. Reference [10] divides the network performance indicators into analytical indicators and experimental indicators, and proposes a protocol-oriented network performance indicator development framework model. This model makes a comprehensive network performance evaluation with relative few indicators, and reduces the number of evaluation indicators effectively. Reference [11] makes network performance evaluation with improved fuzzy AHP algorithm, which is based on triangular fuzzy number analytic hierarchy process and network simulation technology.

This article, for the heterogeneity of the IoT, proposes an application-oriented network performance evaluation model. This model gives the qualitative and quantitative analysis for the IoT performance at the application layer. The heterogeneity of the IoT is shown in Figure 1.

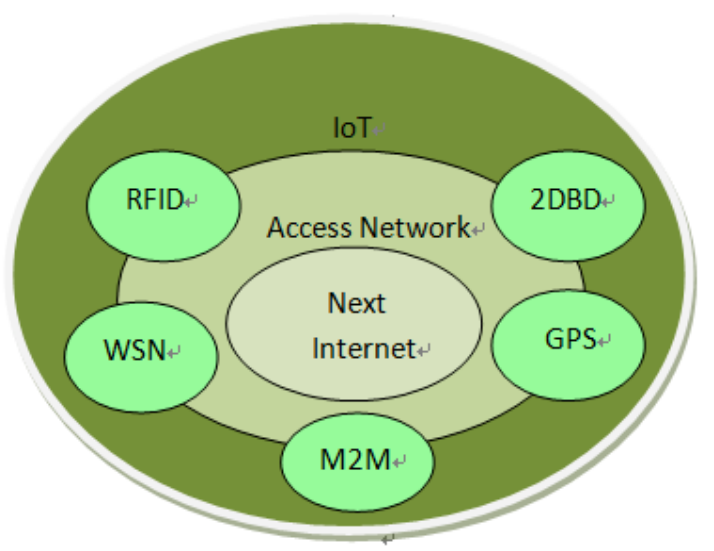

Figure 1. Heterogeneity of the Internet of Things 


\section{DESIGN OF APPLICATION-ORIENTED NETWORK PERFORMANCE EVAluATION MODEL FOR THE INTERNET OF THINGS}

From the application point of view, regardless of the heterogeneity of the bottom layer of the IoT, this paper proposes an application-oriented network performance evaluation model.

\section{A. Network Clustering of the IoT}

According to the difference between the networks at the bottom layer, the IoT can be divided into the following four types of network.

Interactive network: mainly provide interactive services, such as transporting industrial control instruction, database query results, interaction information between radio transmitting and receiving ends, etc. This type of network is commonly used to support online library, remote control systems, unmanned aircraft systems, etc. Interactive network users often require quick and accurate response after sending the command, and it requires that the network delay and data packet delay are as small as possible. Therefore, the evaluation attributes of the interactive network can be response time, packet loss rate and time delay.

Multimedia network: mainly provide multimedia services, such as transporting text, sound, pictures, images and video, etc. This type of network is commonly used to support remote video conference, remote interview, remote education, remote medical treatment, video surveillance systems, etc. Multimedia network users often require large, enough network capacity, and the smaller time delay and time delay jitter. Therefore, the evaluation attributes of the multimedia network can be link utilization, packet loss rate, time delay and time delay jitter.

Data transmission network: mainly provide large-scale data transmission services, such as transporting the conditions and results of the large mathematical calculation, meteorological data, etc. This type of network is commonly used to support large mathematical calculation, weather forecast, etc. Data transmission network users often require data to be obtained is accurate and complete, and the network can deal with sudden, unexpected large amount of data transmission. They allow a certain range of data delay. Therefore, the evaluation attributes of the data transmission network can be network throughput, node throughput, link utilization and packet loss rate.

Wireless network [12] [13] [14]: mainly provide wireless data transmission services, such as wireless transmission of voice, text, instructions, environment information, etc. This type of network is commonly used to support information collection under special environment, logistics systems, supermarket goods management, parking management system, etc. Wireless network users often require the stability and reliability of the network, and the integrity and reliability of the information. Therefore, evaluation attributes of the wireless network can be packet loss rate, time delay and time delay jitter.

\section{B. Qualitative Evaluation Model of Network Performance}

From the application point of view, network performance evaluation attributes are divided into primary network performance evaluation attribute (PNPEA) and secondary network performance evaluation attribute (SNPEA) at the application layer of the IoT.

Primary network performance evaluation attribute: these evaluation attributes are related to the principal activities of the network. The primary evaluation attributes of each network are different, as their main function is different. For example, in real-time network, primary network performance evaluation attributes can be time delay, time delay jitter.

Secondary network performance evaluation attribute: the evaluation attributes can help to improve network performance, but have little relation to the principal activities of the network. Secondary network performance evaluation attributes of each network is different. For example, in an IoT, packet repetition rate can be regarded as a secondary network performance evaluation attribute.

According to primary network performance evaluation attribute and secondary network performance evaluation attribute, network performance can be divided into four levels shown in TABLE I. Color is used to indicate the level of the network performance. Form the best to the worst, they are blue level, yellow level, orange level and red level.

TABLE I.

FOUR LEVELS OF NETWORK PERFORMANCE

\begin{tabular}{|l|l|l|}
\hline level & PNPEA & SNPEA \\
\hline Blue (the best) & $\begin{array}{l}\text { all PNPEA } \\
\text { attributes in the } \\
\text { threshold }\end{array}$ & $\begin{array}{l}\text { all SNPEA } \\
\text { attributes in the } \\
\text { threshold }\end{array}$ \\
\hline Yellow (better) & $\begin{array}{l}\text { all PNPEA } \\
\text { attributes in the } \\
\text { threshold }\end{array}$ & $\begin{array}{l}\text { at least one SNPEA } \\
\text { attribute out of the } \\
\text { threshold }\end{array}$ \\
\hline Orange (worse) & $\begin{array}{l}\text { at least one PNPEA } \\
\text { attribute out of the } \\
\text { threshold }\end{array}$ & $\begin{array}{l}\text { all SNPEA } \\
\text { attributes in the } \\
\text { threshold }\end{array}$ \\
\hline Red (the worst) & $\begin{array}{l}\text { at least one PNPEA } \\
\text { attribute out of the } \\
\text { threshold }\end{array}$ & $\begin{array}{l}\text { at least one SNPEA } \\
\text { attribute out of the } \\
\text { threshold }\end{array}$ \\
\hline
\end{tabular}

\section{Quantitative Evaluation Model of Network} Performance

\section{- Selection of Network Performance Evaluation Attribute}

Before the analysis and evaluation for an IoT, the appropriate evaluation attributes should be selected, according to the results of the cluster analysis in section A, the characteristics of network and users' demands. After selecting evaluation attributes, evaluation attributes values should be calculated with network measurement results.

\section{- Normalization of Network Performance Evaluation Attribute Value}

Before normalization, from the application point of view, the evaluation attributes should be divided basically into two categories. One is upward attribute, and the other is down attribute.

The greater the evaluation attribute value is, the better network performance is on this property, and these attributes are called upward attributes; The smaller the 
evaluation attribute value is, the better network performance is on this property, and these attributes are called down attributes.

The normalization methods of upward attributes and down attributes are different. The normalization method of upward attributes values is shown in (1).

$$
v_{i}=\frac{A V_{i}-T_{i}}{T_{i}} .
$$

In (1), ${ }^{A V}{ }_{i}$ represents attribute value of upward attribute ${ }^{i} ;{ }^{T}{ }^{i}$ represents threshold of upward attribute ${ }^{i}$; represents value of $i$ after normalization.

The normalization method of down attributes values is shown in (2).

$$
v_{i}=\frac{T_{i}-A V_{i}}{T_{i}} .
$$

In (2), ${ }^{A V}{ }_{i}$ represents attribute value of down attribute ${ }^{i} ;{ }^{T}{ }^{i}$ represents threshold of down attribute ${ }^{i}$; represents value of $i$ after normalization.

For some evaluation attributes whose values exceed their threshold range, the evaluation attribute has lost their original significance. After normalization, their values are set to zero.

\section{- Calculation of Network Performance}

\section{Evaluation Value}

A certain period of time is separated as follows: $\left\lfloor t_{1}, t_{2}, t_{3}, \ldots, t_{i}, \ldots, t_{j}, t_{k}, \ldots t_{n}\right\rfloor_{\text {This time can be }}$ divided into equal time segments, and it also can be divided into unequal time segments.

The network performance evaluation value (NPEV) in time segment $\left[t_{i}, t_{j}\right]_{\text {can be calculated as follows. }}$

$$
\begin{aligned}
& V\left(t_{i}, t_{j}\right)=W_{1} * V_{1}+W_{2} * v_{2} \\
& +\ldots+w_{k} * v_{k}+\ldots+w_{n} * v_{n} .
\end{aligned}
$$

The variable $V\left(t_{i}, t_{j}\right)$ represents the network performance evaluation value in time segment $\left[t_{i}, t_{j}\right]_{;} W_{k}$ represents the weight of evaluation attribute $k ; V_{k}$ represents the value of evaluation attribute ${ }^{k}$ after normalization.

Using time $t_{i}$ as the starting time, the cumulative network performance evaluation value (CNPEV) at time $t_{k}$ can be calculated as follows.

$$
L V\left(t_{i}, t_{k}\right)={ }_{W i j} * L V\left(t_{i}, t_{j}\right)+{ }_{W j k} * V\left(t_{j}, t_{k}\right) .
$$

The variable $L V\left(t_{i}, t_{k}\right)$ represents the cumulative network performance evaluation value at the time $t_{k}$. $W_{i j}=\frac{t_{j}-t_{i}}{t_{k}-t_{i}} W_{j k}=\frac{t_{k}-t_{j}}{t_{k}-t_{i}}$.

represents the ratio of the time segment $\left\lfloor t_{i}, t_{j}\right\rfloor$ and the time segment $\left\lfloor t_{i}, t_{k}\right\rfloor$. And ${ }^{W j k}$ is same as ${ }^{W_{i j}}$.

From (4), it can be seen that CNPEV has a memory function. It can record previous network status. NPEV may reflect the operational status of the network in an hour of one day, but CNPEV can reflect the operational status of the network all day.

- Flow Diagram of Application-oriented Network Performance Evaluation Model for the Internet of Things

The flow diagram of application-oriented network performance evaluation model for the Internet of Things is shown in Figure 2. select evaluation

attributes, and determine the primary evaluation attributes and the secondary evaluation attributes
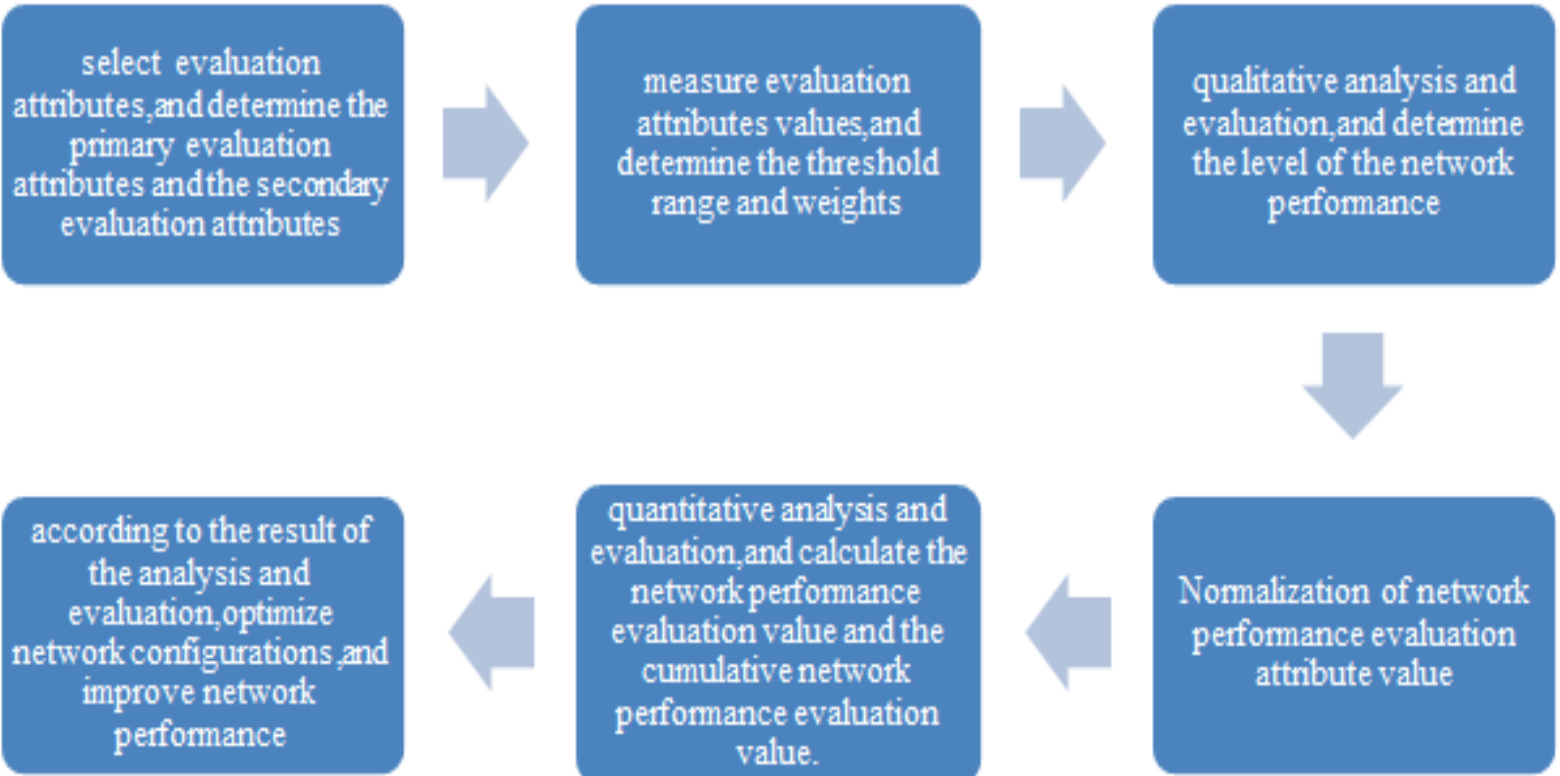

quantitative analysis and evaluation, and calculate the network performance evaluation value and the cumulative network performance evaluation value.

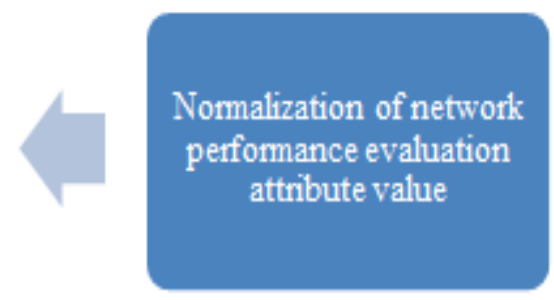

Figure 2. Flow diagram of application-oriented network performance evaluation model for the Internet of Things 


\section{EXPERIMENT}

\section{A. Experiment Environment}

Deploy an IoT platform for environmental monitoring. The bottom layer of this IoT is a wireless sensor network [15], consisting of 22 sensor nodes (one of them is gateway node). The server in the application layer collects data through gateway node and the Internet, then saving the data in the server-side database.

\section{B. Selection of Network Performance Evaluation Attribute}

This IoT platform is mainly used for long-term environmental monitoring, getting reliable and accurate information in the target environment. However, sensor nodes usually carry limited energy. Therefore, it is very necessary to make node load balancing, and make packet repetition low, which can extend the network lifetime effectively. Environmental information to be collected should be accurate and reliable, which requires low packet loss rate. Small $\mathrm{R}$ delay contributes to access to information timely in some special cases. According to the IoT clustering analysis and special application requirements of this IoT, packet loss rate (PLR), packet repetition rate (PRR), node load balancing degree (NLBD), and R delay (RD) are selected as the evaluation attributes of the IoT platform.

From the application point of view, packet loss rate, node load balancing degree, and $\mathrm{R}$ delay belong to primary network performance evaluation attributes; Packet repetition rate belong to secondary network performance evaluation attributes. Packet loss rate, packet repetition rate, and $\mathrm{R}$ delay belong to down attributes; node load balancing degree belongs to upward attributes.

Node load balancing degree: the ratio of the number of the nodes whose load are in the reasonable range and the total number of nodes in this IoT. In this experiment, the reasonable load range is \%: $[1,6]$, and the threshold range of NLBD is \%: [50, 100].

$\mathrm{R}$ delay: the average arriving time of the valid packets received by the terminal at the application layer of the IoT. In this experiment, the threshold range of RD is $\mathrm{s}$ : [0, 30].

In addition, the threshold range of PLR is \%: $[0,50]$; the threshold range of PRR is \%: [0, 10].

\section{Experimental Data}

100,000 data collected form this IoT platform for environmental monitoring was used as experimental data. The data collection time is from 18:00 to 12:00 the next day. Each data consists of node id, parent id, seq, data arrival time, etc. After removing the invalid data and redundant data, the remaining data are divided into three groups in order, and each group has data of 6 hours: [ $\mathrm{t} 1$, $\mathrm{t} 2),[\mathrm{t} 2, \mathrm{t} 3),[\mathrm{t} 3, \mathrm{t} 4)$, and $\mathrm{t} 1=18: 00, \mathrm{t} 2=0.00, \mathrm{t} 3=6: 00$, $\mathrm{t} 4=12: 00$.

\section{Measurement of Evaluation Attribute Value}

Analyze experimental data with data statistical analysis software, IBM SPSS Statistics 20. The results obtained are shown in TABLE II.

TABLE II.

MEASUREMENT RESULTS OF EVALUATION ATTRIBUTE VALUE

\begin{tabular}{|l|l|l|l|l|}
\hline & \multicolumn{1}{|c|}{ PLR } & \multicolumn{1}{c|}{ RR } & \multicolumn{1}{c|}{ NLBD } & \multicolumn{1}{c|}{ RD } \\
\hline$[\mathrm{t} 1, \mathrm{t} 2)$ & $27.0110 \%$ & $17.0932 \%$ & $52.3810 \%$ & $13.4465 \mathrm{~s}$ \\
\hline$[\mathrm{t} 2, \mathrm{t} 3)$ & $86.2678 \%$ & $2.5503 \%$ & $61.9048 \%$ & $76.4929 \mathrm{~s}$ \\
\hline$[\mathrm{t} 3, \mathrm{t} 4)$ & $64 \%$ & $6.2128 \%$ & $66.6667 \%$ & $25.3014 \mathrm{~s}$ \\
\hline threshold & $\%:[0,50]$ & $\%:[0,10]$ & $\%:[50,100]$ & $\mathrm{s}:[0,30]$ \\
\hline weight & 0.3 & 0.1 & 0.3 & 0.3 \\
\hline
\end{tabular}

The threshold range and weights come from experience and application requirements of the IoT platform for environmental monitoring [16].

According to the measurement results and qualitative evaluation model of network performance, a preliminary qualitative evaluation result can be given: Network performance of this IoT platform is at the yellow level in segment $[\mathrm{t} 1, \mathrm{t} 2)$; It is at the orange level in segments $[\mathrm{t} 2$, t3) and [t3, t4). The result shows that network performance of this IoT platform decline overall over time. The node load (number of data packets that nodes transmit) from t1 to $t 4$ can be shown in Figure 3, Figure 4 and Figure 5.

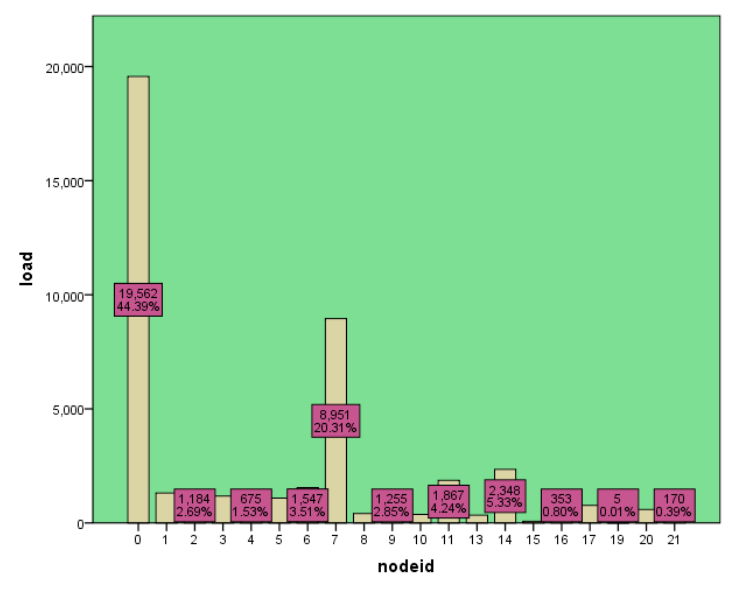

Figure 3. Node load from t1 to t2 


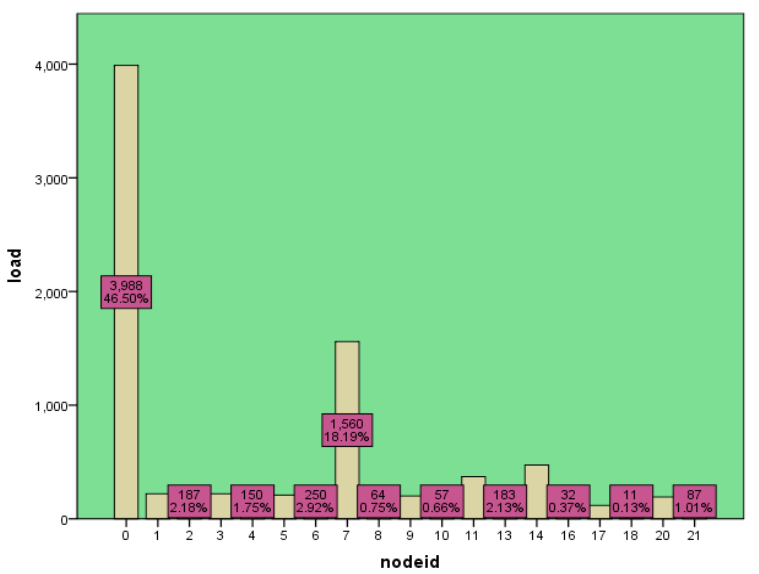

Figure 4. Node load from t2 to $\mathrm{t} 3$

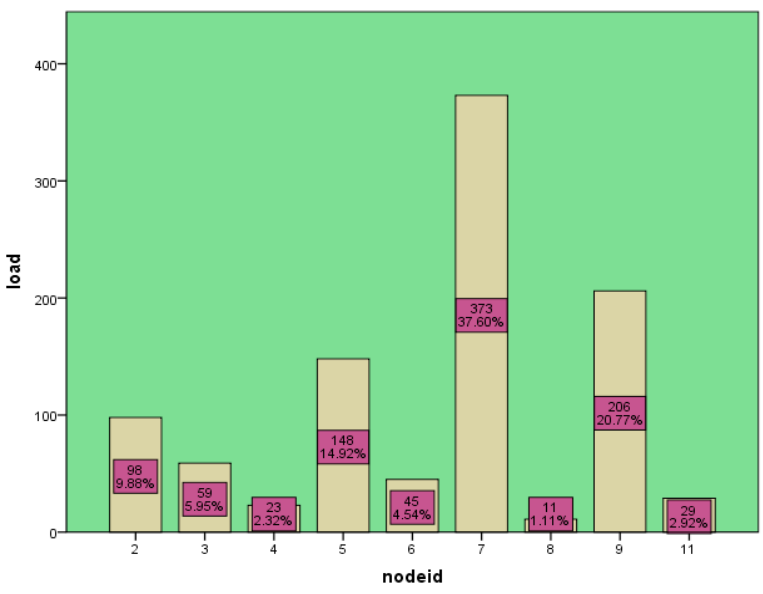

Figure 5. Node load from t3 to t4

\section{E. Experimental Results and Evaluation}

According to quantitative evaluation model of network performance, the results obtained are shown in TABLE III.

TABLE III.

RESULTS OF NEWTORK PERFORMANCE EVALUATION

\begin{tabular}{|l|l|l|}
\hline & NPEV & CNPEV \\
\hline$[\mathrm{t} 1, \mathrm{t} 2)$ & 0.3177 & 0.3177 \\
\hline$[\mathrm{t} 2, \mathrm{t} 3)$ & 0.1459 & 0.2319 \\
\hline$[\mathrm{t} 3, \mathrm{t} 4)$ & 0.1849 & 0.2162 \\
\hline
\end{tabular}

From table 3, the network performance evaluation value (NPEV) is down then up, but it declines overall from the results. The cumulative network performance evaluation value (CNPEV) is not high and declines all the time. This result is consistent with the level of network performance given above.

After analysis, it can be found that the packet loss rate (PLR) in segments $[\mathrm{t} 2, \mathrm{t} 3)$ and $[\mathrm{t} 3, \mathrm{t} 4)$ is so high that the performance of the IoT platform becomes poor. According to the evaluation result, network administrators could optimize network configuration of the IoT platform, reduce PLR, and improve network performance.

\section{CONCLUSIONS}

For the heterogeneity of the IoT, this article proposes application-oriented network performance evaluation model. This model gives the qualitative and quantitative analysis for the performance of the IoT at the application layer, and determines the level of network performance, then calculates the network performance evaluation value and the cumulative network performance evaluation value. From the Experiments Results, it can be known that the network performance level and the two network performance values characterize the status of the Internet of Things accurately. And these evaluation results can provide basis for decision making to optimize network configuration and improve network performance.

\section{ACKNOWLEDGMENT}

This work was supported in part by the National High Technology Research and Development Program of China (Grant No. 2011AA010101), the National Natural Science Foundation of China (Grant No. 61103197 and 61073009), the Science and Technology Key Project of Jilin Province (Grant No. 2011ZDGG007), the Youth Foundation of Jilin Province of China (Grant No. 201101035), and the Fundamental Research Funds for the Central Universities of China (Grant NO.200903179).

\section{REFERENCES}

[1] Luigi Atzori, Antonio lera, Giacomo Morabito, "TheInternet of Things: A survey", in: Computer Networks,2010, 54:2787-2805. http://dx.doi.org/10.1016/j.comnet.2010.05.010

[2] Chen Haiming, Cui Li, Xie Kaibin, "A Comparative Study on Architectures and Implementation Methodologies of Internet of Things", in: CHINESE JOURNAL OF COMPUTERS , 2013,36(1):168-188.

[3] Sun Qibo, Liu Jie, Li Shan, Fan Chunxiao, Sun Juanjuan, "Internet of Things: Summarize on concepts, Architecture and Key Technology Problem", in: Journal of Beijing University of Posts and Telecommunications, 2010, 33(3): 1-9.

[4] Liu Qiang, Cui Li, Chen Haiming, "Key Technologies and Application of Internet of Things", in: Computer Science, 2010, 37(6):1-10.

[5] Jennifer Yick, Biswanath Mukherjee, Dipak Ghosal, "Wireless sensor network survey", in: Computer Networks, 2008, 52:22922330. http://dx.doi.org/10.1016/j.comnet.2008.04.002

[6] Zhang Dongyan, Hu Mingzeng, Zhang Hongli, "Network Performance Evaluation Method Study based on Measurement", in: COMMUNICATIONS, 2006, 27(10): 74-85.

[7] Lei Qing, Wang Xinggang, "Network Performance Evaluation with Network Simulation technology", in: Computer Applications,2001,21(12):11-14.

[8] Meng Rui, Fan Pingzhi, "Research and Implementation of the Evaluation Method of Network Busy", in: Application Research of Computers,2001,(8):41-43.

[9] Guo Rongxiao, Xia Jingbo, Dong Shufu, "Network Performance Comprehensive Evaluation based on Dynamic Empower", in: Microelectronics and Computer, 2011, 28 (5): 48-52.

[10] Zhang Wenjie, Qian Depei, Bai Yuebin, Xu Dawei, Luan Zhongzhi, "A Framework of Network performance evaluation indicator", in: Computer Engineering and Applications,2003:1415.

[11] Tao Yang, Zhang Jing, Zhou Xia, "Network Performance evaluation studies based on triangular fuzzy number analytic hierarchy process and network simulation", in: Computer Applications, 2005, 25(10):2238-2240.

[12] Yuan Lingyun, Wang Xingchao, "Study on Performance Evaluation Method Based on Measurement for Wireless Sensor Network", in: Proceedings of ICCTA 2009 : 201-206. 
[13] Jin-shyan Lee, "Performance Evaluation of IEEE 802.15.4 for Low-Rate Wireless Personal Area Networks", in: IEEE Transactions on Consumer Elect ronics, Vol. 5 2, No. 3, AUGUST 2006:742-749.

[14] Amrita A. Agashe, Dr. S.K. Bodhe, "Performance Evaluation of Mobility Models for Wireless Ad hoc Networks", in: First International Conference on Emerging Trends in Engineering and Technology. IEEE computer society,2008:172-175.

[15] I.F. Akyildiz, W. Su, Y. Sankarasubramaniam, E. Cayirci, "Wireless sensor networks: a survey", in: Computer Networks, 38 (2002): 393-422. http://dx.doi.org/10.1016/S1389-1286(01) $\underline{00302-4}$

[16] ITU-TY. 1540- 2007, Internet protocol data communication service-IP packet transfer and availability performance parameters. ITU, 2007.

\section{AUTHORS}

Xiaobo Yan received the B.S. in College of Computer Science and Technology from Jilin University, China in 2012. He is a Graduate for the M.E. degree with the College of Computer Science and Technology, at Jilin University, Changchun, China. His main research interests include architecture of the Internet of Things, network and information security (e-mail:yanxiaoboccsv@163.com).

Feng Wang received his B.E degree in Information Security in 2009 in Harbin Institute of Technology, followed by his M.E. degree in Network and Information Security in 2012 in Jilin University. Currently, he is a PhD candidate in the College of Computer Science and Technology, Jilin University. His research interests are computer networks, information security, the Internet of Things and Cyber Physical Systems ( e-mail: keepon (a)yeah.net).

Liang Hu received his M.S. and Ph.D. degrees in College of Computer Science and Technology from Jilin University, China in 1993 and 1999. Currently, he is a professor and doctoral supervisor at the College of Computer Science and Technology, Jilin University, China. As an author or co-author, he has published more than 180 research papers, including 80 journal papers, 8 books, and has 1 National invention patent of China as well as 18 computer software copyright registration certificates of China. His research areas are Network Security and Distributed Computing, including theories, models and algorithms of PKI/IBE, IDS/IPS, and Grid Computing. He is a member of the China Computer Federation(e-mail: hul@jlu.edu.cn).

Kuo Zhao received his B.S. degree in Computer Software in 2001 in Jilin University, followed by his M.E. degree in Computer Architecture in 2004 and Ph.D. in Computer Software and Theory from the same university in 2008. Currently, he is an Assoicate Professor in the College of Computer Science and Technology, Jilin University. As an author or co-author, he has published more than 40 research papers, including 15 journal papers, and has 1 National invention patent of China as well as 10 computer software copyright registration certificates of China. His research interests are in operating systems, computer networks and information security ( e-mail: zhaokuo@jlu.edu.cn).

Weiqing Xiong received the B.S. in College of Computer Science and Technology from Jilin University, China in 2013. She has been admitted to Harbin Institute of Technology, studying for the M.E. degree with the School of Computer Science and Technology. Her main research interests include wireless network, network and information security(e-mail: weiqing@yeah.net).

Submitted 16 September 2013. Published as re-submitted by the authors 22 October 2013. 